\title{
Toward an improved wall treatment for multiple-correction k-exact schemes
}

\author{
A. MENASRIA* \\ Ariane Group, 78130 Les Mureaux, FRANCE \\ P. BRENNER ${ }^{\dagger}$ \\ Ariane Group, 78130 Les Mureaux, FRANCE \\ P. CINNELLA \\ Arts et Métiers ParisTech (DynFluid laboratory), 75013 Paris, FRANCE \\ G. $\mathrm{PONT}^{\S}$ \\ Airbus Group, 31700 Blagnac, FRANCE
}

\begin{abstract}
Improved wall boundary treatments are investigated for a family of high-order Godunovtype finite volume schemes based on $k$-exact polynomial reconstructions in each cell of the primitive variables, via a successive corrections procedure. We focus more particularly on the 1-exact and 2-exact schemes which offer a good trade-off between accuracy and computational efficiency. In both cases, the reconstruction stencil needs to be extended to the boundaries. Additionally, information about wall curvature has to be taken into account, which is done by using a surface model based on bicubic Bézier patches for the walls. The performance of the proposed models is presented for two compressible cases, namely the inviscid flow past a Gaussian bump and the viscous axisymmetric Couette flow.
\end{abstract}

\section{Introduction}

LIGH-order finite volume methods for Computational Fluid Dynamics have received considerable interest in recent - 1 years, because of their potential for providing high-fidelity solutions of challenging configurations involving, e.g., flow unsteadiness, boundary layer separation or steep gradients.

A widely studied family of high-order schemes is represented by the so-called $k$-exact schemes, initially introduced by Barth and Frederickson [1] [2] and Vandersbilck and Deconinck [3], which make use of high-order piecewise polynomial reconstructions of the solution and of its derivatives on mesh cells, along with high-order formulae for the computation of flux integrals (see [4] [5] [6] [7] for instance).

In past work [6] [8] [7], a family of $k$-exact schemes ensuring high-accuracy on fully unstructured grids was constructed thanks to an efficient successive corrections procedure. The schemes were applied to the computation of several steady and unsteady flow configurations [7], showing their capability of ensuring high-order accuracy on various grid topologies.

In this context, making sure that these schemes model the flow close to the wall as accurately as possible is of critical importance: indeed, near-wall regions are characterized by several complex phenomena.

Additionally, these exhibit low local values of the Reynolds and Mach numbers. The accuracy of low-Mach flow simulations is directly affected by the ability of compressible solvers to reproduce flows in the incompressible limit [9].

Another important issue for high-order simulations of complex geometries is the treatment of curved boundaries. Approximating a smooth surface (i.e. without ridge or fault) with flat faces introduces a spatial error of order $O\left(h^{2}\right)$, where $h$ is the mesh size [10]. The real shape of the boundary has to be approximated to at least the order of accuracy of the discretization scheme [11].

Finally, an accurate discretization of the boundary conditions is a crucial ingredient for the overall accuracy, although it is not straightforward to achieve.

\footnotetext{
*Ph.D. student, DynFluid/Ariane Group, amandine.menasria@ariane.group

${ }^{\dagger}$ CFD expert, Ariane Group, pierre.brenner@ariane.group

†Professor at Arts et Métiers ParisTech (DynFluid laboratory), AIAA member, paola.cinnella@ensam.eu

${ }^{\S} \mathrm{CFD}$ engineer, Airbus Group, gregoire.pont @ airbus.group
} 
In the $k$-exact schemes proposed in [7] [6] [8], a simplified reconstruction stencil has been used close to solid walls, leading to a local loss of accuracy that can propagate to the entire domain, thus affecting the global order of accuracy of the simulation. Moroever, wall curvature is not taken into account (only polygonal faces have been considered up to now).

In the aim of progressing toward a more accurate representation of flow configurations with curved boundaries, two major improvements of the wall boundary treatment are investigated in the present paper. On the one hand, a high-order representation of wall faces is introduced by means of bicubic Bézier patches, which are then employed to calculate the geometrical moments, that are notably necessary in the fluxes integration in our methodology. On the other hand, solutions to enrich the reconstruction stencil in the vicinity of solid walls in order to improve the local order of accuracy are proposed and analyzed.

All of the present developments are implemented within the industrial code FLUSEPA ${ }^{*}$ developed by the European aerospace company Ariane Group to model all the phases of flight of a space launcher such as takeoff, stage separation, and reentry [12]. The solver can simulate tridimensional, unsteady, compressible, viscous and reactive flows loaded with particles over bodies in relative motion. A CHIMERA-like strategy based on 3D intersections coupled with an Adaptive Mesh Refinement module [13] is used to deal with complex geometries.

The present paper is organized as follow. In Section II the off-wall spatial discretization implemented in FLUSEPA is recalled. The surface model chosen to account for boundary curvature and its implementation within the solver are described in Section III. Strategies for near-wall discretization are presented in Section IV] Numerical validations for selected inviscid and viscous flows are shown and discussed in Section V. Finally, conclusions and perspectives for future work are made in SectionVI

\section{Numerical scheme}

In this Section, the spatial discretization scheme of FLUSEPA [7] [6] is described. Source terms are not considered in the present paper.

Let $q(\boldsymbol{x}, t)$ be a variable verifying a set of conservation equations of the form

$$
\frac{\partial q}{\partial t}+\vec{\nabla} \cdot \boldsymbol{F}=0
$$

$\boldsymbol{F}$ is the flux function of $q$, composed of a convective part that depends only on the solution variable $q$, and of a diffusive part that depends on the solutions variable $q$ and on its gradient $\vec{\nabla} q$.

$$
\boldsymbol{F}=\boldsymbol{F}_{\boldsymbol{c}}(q)+\boldsymbol{F}_{\boldsymbol{d}}(q, \vec{\nabla} q)
$$

The modification of the calculation of diffusive fluxes has not been considered, and will not be detailed in the present article $\left(\boldsymbol{F}_{\boldsymbol{d}}\right.$ is thus assumed null in the following). Note however that improving the accuracy of the solution reconstruction close to walls is also expected to have a beneficial effect on the viscous flux approximation.

In the cell-centered finite volume framework, the domain is divided into $N$ non-overlapping cells $\left(\Omega_{J}\right)_{J \in[|1, N|]}$ of volume $\left|\Omega_{J}\right|$ and cell center $\boldsymbol{x}_{\boldsymbol{j}}=\frac{1}{\left|\Omega_{J}\right|} \iint_{\Omega_{J}} \boldsymbol{x} d V$. The computational variables are the averaged values

$$
\bar{q}_{J}=\frac{1}{\left|\Omega_{J}\right|} \iiint_{\Omega_{J}} q \mathrm{dV}
$$

By integrating Eq. 1 on $\Omega_{J}$ and applying Gauss' theorem, we get

$$
\left|\Omega_{J}\right| \frac{d \bar{q}_{J}}{d t}+\sum_{K=1}^{P} \iint_{A_{J K}} \boldsymbol{F}_{\boldsymbol{c}}(q) \cdot \boldsymbol{n} d S
$$

with $A_{J K}$ the interface between cell $J$ and its neighbor $K, P$ the total number of neighbors of $J$ and $\boldsymbol{n}$ the local outward-pointing normal.

The solver consists in three steps, following the pattern of $k$-exact schemes [1] [2]:

- Reconstruction of the solution in each cell;

- Flux integration along the faces;

- Advance in time.

The time stepping scheme is a second-order Heun scheme with consistent local time stepping (see [8] for details). In the following, time variable $t$ will be omitted to simplify notations.

\footnotetext{
* Registered trademark in France with number 134009261
} 


\section{A. Solution reconstruction}

To reconstruct the solution in a given cell $\Omega_{J}$ to the $k^{\text {th }}$ order, a Taylor expansion of order $k$ at cell centroid $\boldsymbol{x}_{\boldsymbol{j}}$ is performed. For a third-order reconstruction

$$
q(\boldsymbol{x})=q_{j}+\boldsymbol{D}^{(\mathbf{1})} q_{j} \cdot\left(\boldsymbol{x}-\boldsymbol{x}_{j}\right)+\frac{1}{2} \boldsymbol{D}^{(2)} q_{j} \bullet\left[\left(\boldsymbol{x}-\boldsymbol{x}_{j}\right) \otimes\left(\boldsymbol{x}-\boldsymbol{x}_{j}\right)\right]+O\left(h^{3}\right)
$$

$D^{(\mathbf{1})} q$ and $\boldsymbol{D}^{(\mathbf{2})} q$ are respectively the gradient vector and the second derivatives tensor of $q$. Index $j$ refers to pointwise value at $\boldsymbol{x}_{j}$. $\bullet$ is the contracted product and $\otimes$ is the tensor product.

A classical method to determine a reconstruction is to solve the system of Eq. 5 such that the derivatives verify conservativity relations in a least-squares sense [14] [15]. Such method has the drawback to be sensitive to mesh irregularities [16].

Let us call $s(J)$ the direct neighborhood of cell $\Omega_{J}$, comprising the cells $\Omega_{K}$ that share a common edge with $\Omega_{J}$, and $\Omega_{J}$ itself. The reconstruction procedure used in this work is described below.

\section{Linear reconstruction of the solution}

For a second-order accurate reconstruction, we need to calculate $q_{j}$ at second order and $\boldsymbol{D}^{(\mathbf{1})} q_{j}$ at first order.

For this purpose we construct a 1-exact reconstruction operator for the gradient $\mathcal{D}_{\mathbf{1}}^{(\mathbf{1})}[\bar{q}]_{J}$. In this notation:

- the italic font means that the function is a linear approximation operator;

- the index letter refers to the reconstruction stencil. Here it is $J$, hence the operator is defined on $s(J)$;

- $\bar{q}=\left(\bar{q}_{K \in s(J)}\right)$ are the averaged variables of the conservative variables on the stencil $s(J)$. The left and right brackets in $[\bar{q}]$ means that the gradient is constructed as a function of these variables;

- the exponent (1) refers to the derivation order. Here it is first order, which means the operator approximates the first derivatives (solution gradient);

- the index number refers to the accuracy order. Here we are looking for a 1-exact [7] operator on $s(J)$, i.e. such that

$$
\forall \boldsymbol{x} \in s(J), \mathcal{D}_{1}^{(\mathbf{1})}[\bar{q}]_{J}(\boldsymbol{x})=\boldsymbol{D}^{(\mathbf{1})} q(\boldsymbol{x})+O(h) .
$$

In the solver, the reconstruction is applied to the primitive variables since this represents a more robust choice than the conservative ones. For each primitive variable $q$, an average $\tilde{q}$ is defined, which can be expressed as a function of the averaged conservative variables. Reconstruction of $\tilde{q}$ from the conservative variables in the $k$-exact framework is described in [7]. For a second-order accurate reconstruction, we want

$$
\bar{q}=\tilde{q}+O\left(h^{2}\right) .
$$

To compute the gradient, a technique based on a modification of the Green-Gauss method is used [7] [8]. Gauss' theorem is applied to the exact gradient to bring back the problem to the calculation of surface integrals, which are then approximated by using a linear interpolation between the left and right averaged cells values.

$$
\begin{gathered}
\frac{1}{\left|\Omega_{J}\right|} \iiint_{\Omega_{J}} \boldsymbol{D}^{(\mathbf{1})} q(\boldsymbol{x}) d V=\frac{1}{\left|\Omega_{J}\right|} \sum_{K=1}^{P} \iint_{A_{J K}} q \boldsymbol{n} d S=\boldsymbol{D}^{(\mathbf{1})} q\left(\boldsymbol{x}_{\boldsymbol{j}}\right)+O(h) \\
\iint_{A_{J K}} q \boldsymbol{n} d V=\left(\beta_{K} \bar{q}_{K}+\left(1-\beta_{K}\right) \bar{q}_{J}\right) \boldsymbol{A}_{J K}, \text { with } \beta_{K}=\frac{\left\|\boldsymbol{x}_{\boldsymbol{j}}-\boldsymbol{x}_{\boldsymbol{\Gamma}}\right\|}{\left\|\boldsymbol{x}_{\boldsymbol{j}}-\boldsymbol{x}_{\boldsymbol{\Gamma}}\right\|+\left\|\boldsymbol{x}_{\boldsymbol{k}}-\boldsymbol{x}_{\boldsymbol{\Gamma}}\right\|} \in[0,1]
\end{gathered}
$$

with $\boldsymbol{A}_{\boldsymbol{J} \boldsymbol{K}}=\boldsymbol{O}\left(h^{2}\right) \boldsymbol{n}_{\boldsymbol{J} \boldsymbol{K}}$ the surface vector of interface $A_{J K},\left|\Omega_{J}\right|=O\left(h^{3}\right)$ the volume of cell $J$, and $\boldsymbol{x}_{\boldsymbol{\Gamma}}$ a chosen point related to the interface called integration poin Note that this formula is not used for wall faces: the original solver uses $\beta_{K}=0$ for these faces such that the contribution of the wall is totally excluded from the recontruction stencil. This will be modified in Section IV

Additional conditions for the 1-exact gradient operator are given by the $k$-exact approach

$$
\left\{\begin{array}{l}
\sum_{K \in s(J)} \boldsymbol{W}_{\boldsymbol{J} K}=\mathbf{0}(0 \text {-exactness }) \\
\sum_{K \in s(J)}\left(\boldsymbol{\alpha} \cdot\left(\boldsymbol{x}_{\boldsymbol{k}}-\boldsymbol{x}_{\boldsymbol{j}}\right)\right) \boldsymbol{W}_{\boldsymbol{J} \boldsymbol{K}}=\boldsymbol{\alpha}(1 \text {-exactness }) .
\end{array}\right.
$$

\footnotetext{
${ }^{\dagger}$ The choice of such a point will be discussed in Section III
} 
where $\left(\boldsymbol{W}_{\boldsymbol{J} K}\right)_{K \in s(J)}$ are the weight vectors such that $\mathcal{D}_{1}^{(\mathbf{1})}[\bar{q}]_{J}=\sum_{K \in S(J)} \boldsymbol{W}_{\boldsymbol{J}} \bar{q}_{K}$.

By combining equations 8 and 9 , we obtain a first discrete approximation for the gradient given by

$$
\mathcal{D}_{\mathbf{0}}^{(\mathbf{1})}[\bar{q}]_{J}=\frac{1}{\left|\Omega_{J}\right|} \sum_{K \in S(J)}\left(\beta_{K} \bar{q}_{K}+\left(1-\beta_{K}\right) \bar{q}_{J}\right) \boldsymbol{A}_{J K}
$$

However, if approximation 9 is of order $O\left(h^{2}\right) \boldsymbol{A}_{\boldsymbol{J} \boldsymbol{K}}$ when $\boldsymbol{x}_{\boldsymbol{j}}, \boldsymbol{x}_{\boldsymbol{\Gamma}}$ and $\boldsymbol{x}_{\boldsymbol{k}}$ are aligned, it is only of order $\boldsymbol{O}(h) \boldsymbol{A}_{\boldsymbol{J} \boldsymbol{K}}$ otherwise. Thus the gradient operator 11 is no more consistent on general meshes. To recover consistency on unstructured grids, a corrective operator is built, with its associated matrix $M_{1}(J)$ called simple correction matrix [8]. The matrix $M_{1}(J)$ of the linear operator 11 is established by applying the conditions 10 to the canonical basis of the polynomials of degree $1\left(x-x_{j}, y-y_{j}, z-z_{j}\right)$. We get

$$
M_{1}(J) \mathcal{D}_{1}^{(1)}[\bar{q}]_{J}=\mathcal{D}_{\mathbf{0}}^{(\mathbf{1})}[\bar{q}]_{J}
$$

and finally obtain what we call a Quasi-Green 1-exact gradient operator

$$
\mathcal{D}_{\mathbf{1}}^{(\mathbf{1})}[\bar{q}]_{J}=M_{1}^{-1}(J) \frac{1}{\left|\Omega_{J}\right|} \sum_{K \in S(J)}\left(\beta_{K} \bar{q}_{K}+\left(1-\beta_{K}\right) \bar{q}_{J}\right) \boldsymbol{A}_{J K}, \text { with } M_{1}(J)=\sum_{K \in S(J)} \beta_{K} \boldsymbol{A}_{\boldsymbol{J}} \otimes\left(\boldsymbol{x}_{\boldsymbol{k}}-\boldsymbol{x}_{\boldsymbol{j}}\right)
$$

and $M_{1}^{-1}(J)$ the inverse of matrix $M_{1}(J)$. Note that for cartesian meshes, $M_{1}(J)$ is the identity matrix.

This procedure leads a second-order accurate scheme.

\section{Parabolic reconstruction of the solution}

For a third-order accurate reconstruction we need to calculate $q_{j}$ at third order, $\boldsymbol{D}^{(\mathbf{1})} q_{j}$ at second order and $\boldsymbol{D}^{(\mathbf{2})} q_{j}$ at first order.

First, we must compute the primitive averaged values $\tilde{q}$ with third-order accuracy. A correction term $\Delta q$ can be established using what has been done in part 1] details of this procedure can be found in [7].

Afterwards, successive corrections of the derivatives are carried out. The 1-exact gradient operator described above is applied twice, leading to an inconsistent approximation of the second derivatives in $\boldsymbol{x}_{\boldsymbol{j}}$

$$
\mathcal{D}_{0}^{(2)}[\bar{q}]_{J}=\mathcal{D}_{1}^{(1)}\left[\mathcal{D}_{1}^{(1)}[\bar{q}]_{J}\right]_{K}
$$

The consistency of the second-order accurate gradient operator is restored on general meshes using the linearity properties of the numerical gradient operator and 2-exactness constraints (see [17]). This gives the third-order tensor $M_{2}(J)$, which depends on geometrical quantities of two levels of neighbors of cell $J$ (neighbors of $J$ and their neighbors).

$$
\mathcal{D}_{1}^{(2)}[\bar{q}]_{J}=M_{2}(J)^{-1} \mathcal{D}_{0}^{(2)}[\bar{q}]_{J}
$$

We then correct the 1-exact approximation gradient to increase its accuracy to the second order, by calculating the truncation error between the exact gradient and its 1-exact reconstruction operator. For each component $m \in\{1,3\}$ of the gradient, a matrix $H_{1}(J)_{m}$ is calculated, which depends only on geometrical quantities of the cells of the direct neighborhood $s(J)$ :

$$
\left(\boldsymbol{D}^{(\mathbf{1})} q_{j}\right)_{m}=\overbrace{\left(\mathcal{D}_{\mathbf{1}}^{(\mathbf{1})}[\bar{q}]_{J}\right)_{m}-\boldsymbol{D}^{(\mathbf{2})} q_{j} \bullet H_{1}(J)_{m}}^{\left(\mathcal{D}_{\mathbf{1}}^{(\mathbf{1}}[\bar{q}]_{J}\right)_{m}}+O\left(h^{2}\right)
$$

In $16, D^{(2)} q_{j}$ is established using the approximation 15

This procedure leads to a third-order scheme. 


\section{B. Flux integration}

Flux integration along face $A_{J K}$ is achieved by using a one-point integration formula, based on a Taylor expansion to the third order:

$$
\iint_{A_{J K}} \boldsymbol{F}_{\boldsymbol{c}} \cdot \boldsymbol{n} d S=\boldsymbol{F}_{c \mid \Gamma} \cdot \boldsymbol{S}_{A_{J K}}^{(0)}+\boldsymbol{D}^{(1)} \boldsymbol{F}_{c \mid \Gamma} \cdot \mathcal{S}_{A_{J K}}^{(1)}+\frac{1}{2} \boldsymbol{D}^{(2)} \boldsymbol{F}_{c \mid \Gamma} \bullet \mathcal{S}_{A_{J K}}^{(2)}+O\left(h^{3}\right) \boldsymbol{A}_{\boldsymbol{J} \boldsymbol{K}}
$$

where $\boldsymbol{x}_{\boldsymbol{\Gamma}}$ is the integration point and where $\mathcal{S}_{\boldsymbol{A}_{\boldsymbol{J}}}^{(\boldsymbol{m})}$ are the surface moments of order $m$ of the interface $A_{J K}$

$$
\mathcal{S}_{\boldsymbol{A}_{\boldsymbol{J} K}}^{(\boldsymbol{m})}=\iint_{A_{J K}}\left(\boldsymbol{x}-\boldsymbol{x}_{\Gamma}\right)^{\otimes m} \boldsymbol{n} d S, m \in\{0,1,2\}
$$

Surface moments are fundamental for the solver. They are tensors of order $m$ which are sized in $O\left(h^{m}\right)$. As will be seen in Section [III, particular attention must be paid to the calculation of surface moments on the one hand, and on the choice of the integration point on the other hand when taking into account curvature.

Eq. 17]is different from the classical choice of Gauss points to integrate fluxes. For a third-order reconstruction process, we need $\boldsymbol{F}_{\boldsymbol{c} \mid \boldsymbol{\Gamma}}$ at order $3, \boldsymbol{D}^{(\mathbf{1})} \boldsymbol{F}_{\boldsymbol{c} \mid \boldsymbol{\Gamma}}$ at order 2 and $\boldsymbol{D}^{(\mathbf{2})} \boldsymbol{F}_{\boldsymbol{c} \mid \boldsymbol{\Gamma}}$ at order 1. These approximations are obtained by solving a Riemann problem at the interface $A_{J K}$. Left and right values of the variable are given by the solution reconstruction and derivative approximates established previously, following a MUSCL-type approach [18].

Note that the overall $k$-exact scheme presented in this Section can formally be derived to any order of accuracy. For a scheme of order $m$, one would have to perform the fluxes' expansion to the $m^{t h}$ order, and to derive the corresponding adapted approximations of the derivatives using the successive corrections procedure generalized from what has been done for the 2-exact reconstruction scheme.
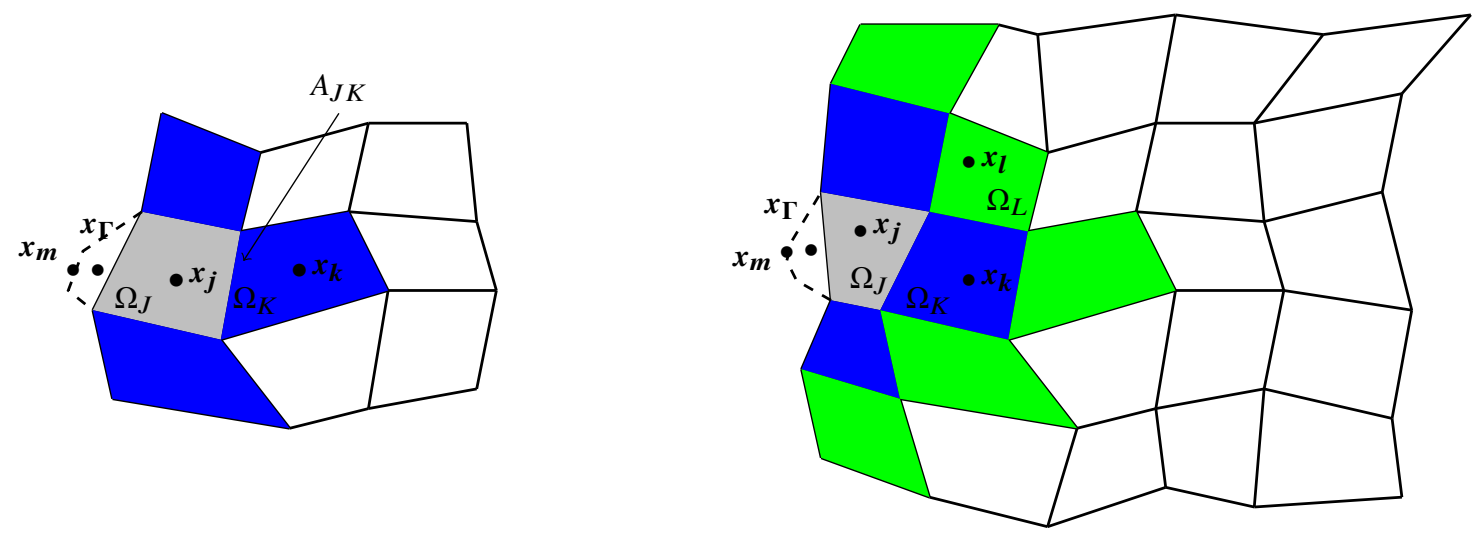

Fig. 1 Discretization stencil at the wall and curved wall face for the: (a) 1-exact reconstruction scheme; (b) 2-exact reconstruction scheme

III. Wall curvature

Modelling the wall curvature is a prerequisite to high-order boundary treatment. In the baseline implementation of FLUSEPA, boundary faces are represented by flat face 7 This introduces a second-order spatial error compared to the actual surface [10]. However, the approximation of the physical surface has to be at least of the order of the numerical scheme to ensure the nominal order of accuracy close to the wall [11]. In the case of parametric surfaces using a polynomial basis, the polynomials have to be at least of degree two.

For this purpose, we use the geometric module BSHAPE developed by INRIA [19], which generates from a surface mesh a composite surface made of bicubic Bézier patches.

A bicubic Bézier patch is a parametric polynomial surface of degree three originated by De Casteljau and Bézier [20] around the same time. Given a set of 16 control points $\left(\boldsymbol{P}_{i j}\right)_{i \in\{0,1,2,3\}, j \in\{0,1,2,3\}}$, the equation of the Bézier patch is given by [21]:

\footnotetext{
${ }^{\ddagger}$ More precisely, triangular faces are used. Quadrangular faces are treated by being split into two triangles.
} 


$$
\forall(u, v) \in[0,1] \times[0,1], \boldsymbol{f}(u, v)=\sum_{i=0}^{3} \sum_{j=0}^{3} B_{i}^{3}(u) B_{j}^{3}(v) \boldsymbol{P}_{i j}
$$

where $\forall u \in[0,1], \forall i \in\{0,1,2,3\}, B_{i}^{3}(u)=\frac{3 !}{i !(3-i) !}(1-u)^{3-i} u^{i}$ are the Bernstein polynomials of degree three.

BSHAPE module is based on a point normal interpolation meshing strategy [22]: the patches are generated with the points coordinates and associated normals of the mesh. The modelization ensures G1 continuity, i.e. tangent plane continuity, between the patches.

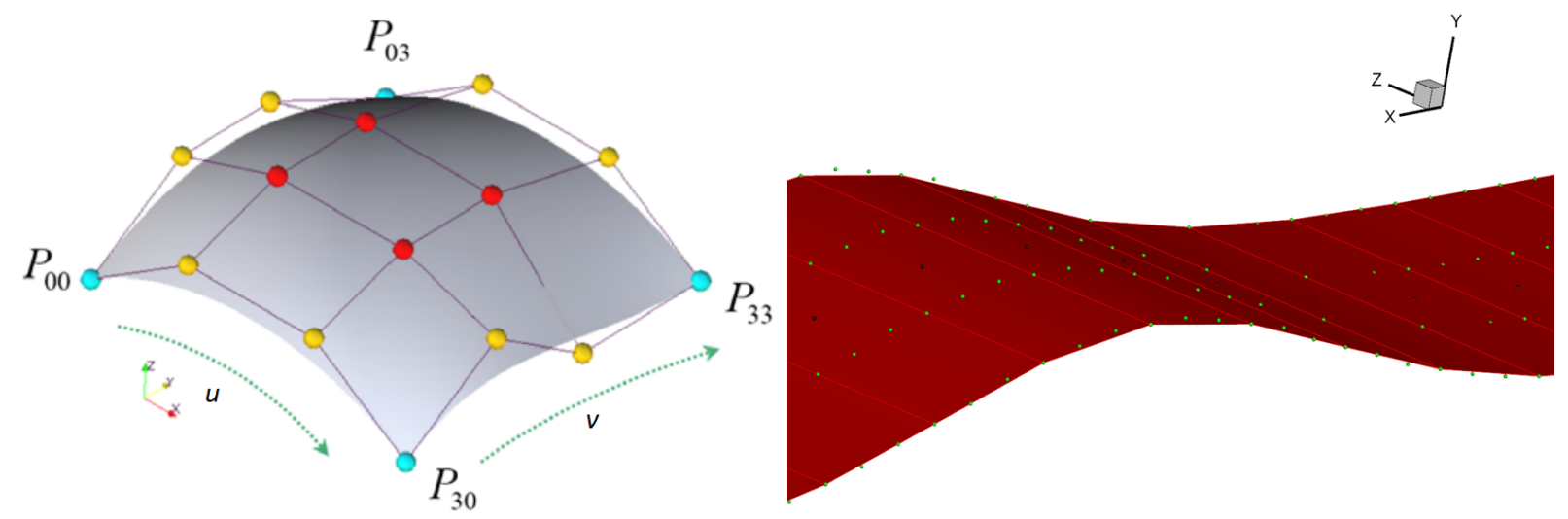

Fig. 2 (a) Representation of a Bézier patch; (b) Control points $\left(\boldsymbol{P}_{i, j}\right)_{i \in[\mid 1,3], j \in[11,3 \mid]}$ and middle point $\boldsymbol{x}_{\boldsymbol{m}}$ generated by the solver for the wall faces of the bump test case

The module has been incorporated in FLUSEPA. The geometry routine selects the wall faces and the side faces sharing an edge with the wall faces, and generates a set of control points corresponding to a patch for each wall face (see Figure 2). The composite surface is not directly seen by the solver, that is, a new mesh is not created, but the information about the patches, namely the control points, is used to integrate the boundary geometric quantities required for the scheme introduced in Section III The surface moments of the wall faces are derived directly by integrating analytically the constitutive equation of the patch. Then volume moments and centroids of the boundary cells are deduced by the application of Gauss' theorem.

This strategy was tested on simple configurations for which the analytical equation of the surface is known (parabolic channel, sphere). Geometrical quantities (such as the surface and volume moments required by the $k$-exact scheme) were calculated using 19 and we observed that the error decreased from 1 up to 2 orders of magnitude when the module was used.

In addition to computing more accurately the geometrical quantities, flux integration on curved wall faces needs further modifications.

In the original solver, the integration point $\boldsymbol{x}_{\boldsymbol{\Gamma}}$ is constructed to minimize the first-order error in the flux integration: $\boldsymbol{x}_{\boldsymbol{\Gamma}}$ is chosen so that the associated surface moments of order 1 are orthogonal to $\boldsymbol{A}_{\boldsymbol{J} \boldsymbol{K}}$. Consequently, the first-order error in the normal face direction $\boldsymbol{n}_{\boldsymbol{J}}=\frac{\boldsymbol{A}_{\boldsymbol{J}}}{\left\|\boldsymbol{A}_{\boldsymbol{J} K}\right\|}$ cancels out by construction.

$$
\boldsymbol{x}_{\boldsymbol{\Gamma}}=\left(\begin{array}{l}
\frac{1}{\left\|A_{J K}\right\|}\left(\iint_{A_{J K}} x \boldsymbol{n} d S\right) \cdot \boldsymbol{n}_{\boldsymbol{J} K} \\
\frac{1}{\left\|A_{J K}\right\|}\left(\iint_{A_{J K}} y \boldsymbol{n} d S\right) \cdot \boldsymbol{n}_{\boldsymbol{J} K} \\
\frac{1}{\left\|A_{J K}\right\|}\left(\iint_{A_{J K}} z \boldsymbol{n} d S\right) \cdot \boldsymbol{n}_{\boldsymbol{J} K}
\end{array}\right)
$$

In case of a flat face, $\boldsymbol{n}$ is constant equal to $\boldsymbol{n}_{\boldsymbol{J} \boldsymbol{K}}$ thus $\boldsymbol{x}_{\boldsymbol{\Gamma}}$ is the center of gravity of the face and lies on the face. However, when the face is curved, $\boldsymbol{n}$ is not constant: $\boldsymbol{x}_{\boldsymbol{\Gamma}}$ is not equal a priori to the center of gravity of the face. More importantly, it does not generally lie on the face.

In case of a curved wall, flux and its derivatives needed in 17 are evaluated at a particular point of the Bézier patch. In a first approach, the middle point of the patch $\boldsymbol{x}_{\boldsymbol{m}}$ is chosen (point of the patch with parameters $u=0.5$ and $v=0.5$ ). This is not a problem for the error generated during the flux integration, as long as the flux and its derivatives are 
approximated to the required order of accuracy. Concerning the resolution of the Riemann problem at the wall, the local normal at point $\boldsymbol{x}_{\boldsymbol{m}}$ is used instead of the integrated normal $\boldsymbol{n}_{\boldsymbol{J} \boldsymbol{K}}$.

The radius of curvature is also computed for each patch and will be used in Section IV]in the boundary conditions of Euler cases. In two-dimensional space, any twice differentiable curve can be locally approximated by a circular arc whose radius is called the radius of curvature. In three-dimensional space, several types of curvatures can be defined. In the present work, we consider the normal curvature associated to a given direction. The tangential velocity (generally non-zero for Euler cases) is calculated at the middle point of the patch, and the radius of curvature $R$ associated to this vector and to the normal at this point is estimated (see Chapter 19 of [21]).

\section{Near-wall discretization}

In this Section, a near-wall discretization consistent with the second and third-order accurate schemes described in Section II is derived. FLUSEPA originally does not take into account the wall in the polynomial reconstruction of the solution variable: for wall faces, the parameter $\beta$ introduced in Section $\mathrm{II}$ is set to 0 , which corresponds to a complete decentering to the inside of the domain.

The original stencils used respectively for the second and third-order schemes corresponding to a boundary cell $\Omega_{J}$ are shown in Figure 1.

In the following, we want to account for the wall contribution in the calculation of the derivatives approximation operators. Denote by $\Omega_{K_{0}}$ the wall boundary face of a given cell $\Omega_{J}, A_{J K_{0}}$. This would correspond to a volumeless cell and is actually just a notation that will permit to extend formally the previous formulae. Let us denote $s^{*}(J)$ the extended neighborhood of $\Omega_{J}$ as

$$
s^{*}(J)=s(J) \cup \Omega_{K_{0}}
$$

with the convention that $\Omega_{K_{0}}=\emptyset$ when $\Omega_{J}$ is not a boundary cell, so that the extended stencil $s^{*}(J)$ is equal to $s(J)$ when $\Omega_{J}$ is inside the domain, and symmetrizes the stencil when $\Omega_{J}$ is a boundary cell.

The extension of the numerical scheme is explained hereafter for the 1-exact and for the 2-exact reconstructions of the solution variables. Treatment of the wall boundary conditions for the Euler and Navier-Stokes equations is also discussed.

\section{A. Modification of the linear reconstruction for boundary cells}

In this part, a simple approach for achieving a truly second-order accurate reconstruction of the solution in boundary cells is presented.

To build a second-order polynomial representation of the variable $q$ that uses the wall information, the Quasi-Green gradient is modified consistently with the methodology introduced in the first part of Section II Assume that a Dirichlet condition function $q_{D}(\boldsymbol{x} \in \partial \Omega)$ is prescribed at the wall. We need to find a value at the wall $q_{k_{0}}$ such that, similarly to Eq. 8

$$
\iint_{A_{J} K_{0}} q(\boldsymbol{x}) \boldsymbol{n}(\boldsymbol{x}) d S \approx\left(\beta_{K_{0}} q_{k_{0}}+\left(1-\beta_{K_{0}}\right) \bar{q}_{J}\right) \boldsymbol{A}_{\boldsymbol{J} \boldsymbol{K}_{\mathbf{0}}}
$$

A Taylor expansion of $q_{D}$ at any point of the wall face $\boldsymbol{x}_{\boldsymbol{W}} \in A_{J K_{0}}$ in the integral of 22 gives:

$$
\iint_{A_{J} K_{0}} q(\boldsymbol{x}) \boldsymbol{n}(\boldsymbol{x}) d S=\iint_{A_{J K_{0}}}\left(q_{D}\left(\boldsymbol{x}_{\boldsymbol{W}}\right)+\boldsymbol{O}(h)\right) \boldsymbol{n} d S, \text { with } \boldsymbol{x}_{\boldsymbol{W}} \in A_{J K_{0}} .
$$

thus:

$$
\iint_{A_{J K_{0}}} q \boldsymbol{n} d S=q_{D, W} \boldsymbol{A}_{\boldsymbol{J} K_{\mathbf{0}}}+\boldsymbol{O}(h) \boldsymbol{A}_{J K_{\mathbf{0}}}
$$

and finally:

$$
\iint_{A_{J K_{0}}} q \boldsymbol{n} d S=\left(\beta_{K_{0}} q_{D, W}+\left(1-\beta_{K_{0}}\right) \bar{q}_{J}\right) \boldsymbol{A}_{\boldsymbol{J} \boldsymbol{K}_{\mathbf{0}}}+\boldsymbol{O}(h) \boldsymbol{A}_{\boldsymbol{J} \boldsymbol{K}_{\mathbf{0}}} \text { with } \beta_{K_{0}}=1
$$


This wall contribution approximation is only first-order accurate on general grids, however this is not a problem since by construction, the consistency of the overall gradient operator will be restored thanks to the simple correction matrix $M_{1}(J)$. More precisely, $M_{1}(J)$ must be modified accordingly to 25 in order to satisfy the 1-exactness consistency condition of Eq. 10. By following the same reasoning as in Eq. 13, we obtain the simple correction matrix with a reconstruction stencil extended to the wall, noted $M_{1}^{*}(J)$ :

$$
M_{1}^{*}(J)=\frac{1}{\left|\Omega_{J}\right|} \sum_{K \in s^{*}(J)} \beta_{K} \boldsymbol{A}_{\boldsymbol{J} K} \otimes\left(\boldsymbol{x}_{\boldsymbol{k}}-\boldsymbol{x}_{\boldsymbol{j}}\right), \text { with } \beta_{K_{0}}=1 \text { and } \boldsymbol{x}_{\boldsymbol{K}_{\mathbf{0}}}=\boldsymbol{x}_{\boldsymbol{W}}
$$

Finally, a new 1-exact gradient operator $\mathcal{D}_{1}^{*(\mathbf{1})}$ based on a more symmetrical stencil is proposed

$$
\mathcal{D}_{1}^{*(\mathbf{1})}[\bar{q}]_{J}=M_{1}^{*-1}(J) \frac{1}{\left|\Omega_{J}\right|} \sum_{K \in s^{*}(J)}\left(\beta_{K} \bar{q}_{K}+\left(1-\beta_{K}\right) \bar{q}_{J}\right) \boldsymbol{A}_{J K}
$$

with the notation $\bar{q}_{K_{0}}=q_{D, W}$.

Let's now consider the case for which a Neumann condition $\frac{\partial q}{\partial n}(\boldsymbol{x} \in \partial \Omega)=\boldsymbol{D}^{\mathbf{1}} \boldsymbol{q}(\boldsymbol{x}) \cdot \boldsymbol{n}(\boldsymbol{x})=q_{N}(\boldsymbol{x})$ is prescribed at the boundary. To avoid multiple definitions of the simple correction matrix $M_{1}$, a second-order accurate equivalent fictive value at the wall $q_{W}^{f i c t}$ is calculated in order to fall back into the Dirichlet case.

However, one has to be careful to evaluate this fictive value at a unique point $\boldsymbol{x}_{\boldsymbol{W}}$, independently on the type of boundary condition. For Dirichlet-type conditions, we have seen before that any point could be chosen for $\boldsymbol{x}_{\boldsymbol{W}}$, as long as it lies on the wall face. For Neumann-type conditions, we perform a Taylor expansion in the normal direction of the chosen wall point to obtain this fictive value. Hence, our choice is to choose the wall point as the point $\boldsymbol{x}_{\boldsymbol{W}}=\boldsymbol{x}_{\boldsymbol{N}}$ whose corresponding normal is colinear to the vector $\boldsymbol{x}_{N}-\boldsymbol{x}_{\boldsymbol{j}}$. In these conditions, $q_{W}^{f i c t}$ is given by

$$
q_{W}^{f i c t}=\bar{q}_{J}-\frac{\partial q}{\partial n} \mid W\left(\left(\boldsymbol{x}_{\boldsymbol{j}}-\boldsymbol{x}_{\boldsymbol{W}}\right) \cdot \boldsymbol{n}_{\boldsymbol{W}}\right)+\boldsymbol{O}\left(h^{2}\right)
$$

\section{B. Modification of the parabolic reconstruction for boundary cells}

As can be seen in Figure 1 (b),the Quasi-Green operator $\mathcal{D}_{1}^{*(\mathbf{1})}[\bar{q}]_{J}$ established in part $\mathrm{A}$ can not be applied twice since gradients in the neighborhood of $\Omega_{J}$ are missing (assuming a Dirichlet-type boundary condition).

We choose to apply the enriched gradient operator and its associated correction matrix whenever possible, and to keep the baseline approximation otherwise. With this choice, the initial (inconsistent) approximation of the second derivatives is obtained by applying the modified 1-exact operator $\mathcal{D}_{1}^{*(\mathbf{1})}$ onto the original operator $\mathcal{D}_{1}^{(\mathbf{1})}$ :

$$
\mathcal{D}_{0}^{*(2)}[\bar{q}]_{J}=\mathcal{D}_{1}^{(1)}\left[\mathcal{D}_{1}^{*(1)}[\bar{q}]_{J}\right]_{K}
$$

The correction matrix $M_{2}(J)$ is modified in a similar way and a matrix $M_{2}^{*}(J)$ is derived.

Then, the geometrical matrices $\left(H_{1}(J)\right)_{i \in\{1,3\}}$ appearing in Eq. 16 which give the truncation error between the exact gradient and its 1-exact representation are changed using what has been done in paragraph $\mathrm{A}\left(H_{1}(J)\right)_{i \in\{1,3\}}$ originally depends on the parameters $\beta_{K}$ and on the second-order volume moments of the cells of $s(J)$. We thus take in the wall contribution $\beta_{K_{0}}=1$ and the volume moments for cell $\Omega_{K_{0}}$ null to calculate $\left(H_{1}^{*}(J)\right)_{i \in\{1,3\}}$.

The delicate point of this method is the accuracy of the boundary value $q\left(\boldsymbol{x}_{\boldsymbol{W}}\right)$. Indeed, thanks to the correction matrices, the derivatives will reach their expected order of accuracy in boundary cells (second-order for the gradient, first order for the second derivatives) if and only if $q_{W}$ is at least third-order accurate. Boundary conditions for $q_{W}$ are studied in the next paragraph.

In part A, we chose to directly make the surface integral of Eq. 25 (which corresponds to the wall contribution of the operator 12 consistent, which led us to $\beta_{W}=1$, and then we used the extended 1-exact gradient in the successive corrections procedure. Other approaches are possible. For example, a reasoning on the global method in one dimension shows that $\beta_{W}=3$ enables a direct obtention of a second-order accurate gradient at the boundary cell centroid (even if the surface integral is no more consistent). In three dimensions this method is a bit more complicated: choosing a $\beta_{W}$ such that the truncation error of order 1 cancels out is not straightforward, since the system to solve is non-linear, due to the inverse of the $M_{1}^{*}(J)$ matrix which depends also on $\beta_{W}$. An idea is to linearize the system and to project it in a privileged direction (normal direction for instance) in which the first-order error on the approximation gradient will cancel out. In all cases, the prerequisite for a truly $k$-exact reconstruction when $\beta_{W} \neq 0$ is to approximate the pointwise value at the wall point at least to $O\left(h^{n+1}\right)$. 


\section{Boundary conditions}

In the previous parts, the reconstruction stencil of the $k$-exact schemes of order 2 and 3 have been extended for boundary cells, using the information available at the wall through boundary (Dirichlet and Neumann) conditions. An implicit assumption was made that these boundary conditions were known exactly at the wall. In practice, this is not generally the case. In the following, proper approximations of the physical wall boundary conditions are investigated for the Euler and Navier-Stokes equations.

For the scheme with 1-exact reconstruction of the solution, pointwise boundary values of the variables at $\boldsymbol{x}_{\boldsymbol{W}}$ must be known to the second order of accuracy.

For the Euler equations, a slip condition at the wall is prescribed for the velocity:

$$
\forall x \in A_{J K_{0}}, u(x) \cdot n(x)=u_{m e s h}
$$

with $\boldsymbol{u}_{\boldsymbol{m} \boldsymbol{m} \boldsymbol{s} \boldsymbol{h}}$ the wall velocity.

Hence, if a Dirichlet-type information is available on the normal velocity, no information is a priori known about the tangential velocity at the wall. This problem is likely to deteriorate the accuracy of the gradient approximation. An apparent solution to calculate $\boldsymbol{u}_{\boldsymbol{W}}$ would be to remove its normal part to the extrapolated value of the velocity in the boundary cell: $\boldsymbol{u}_{\boldsymbol{W}}=\overline{\boldsymbol{u}}_{\boldsymbol{J}}-\overline{\boldsymbol{u}}_{\boldsymbol{J}} \cdot \boldsymbol{n}_{J K}$. However, this approximation for the velocity at the wall is at most of order one.

A way of dealing with this issue is to use the computed velocity and velocity gradients of the boundary cell at the previous time to correct the wall velocity value:

$$
\boldsymbol{u}_{\boldsymbol{W}_{\mathbf{0}}}=\overline{\boldsymbol{u}}_{\boldsymbol{J}}+\mathcal{D}_{\mathbf{1}}^{*(\mathbf{1})}[\overline{\boldsymbol{u}}]_{J} \cdot\left(\boldsymbol{x}_{\boldsymbol{W}}-\boldsymbol{x}_{\boldsymbol{j}}\right)+O\left(h^{2}\right)
$$

where the right-hand side of this equation is taken at the previous timestep; and then to remove the normal part of this value

$$
\boldsymbol{u}_{\boldsymbol{W}}=\boldsymbol{u}_{\boldsymbol{W}_{0}}-\left(\boldsymbol{u}_{W_{0}} \cdot \boldsymbol{n}_{\boldsymbol{W}}\right) \boldsymbol{n}_{\boldsymbol{W}}
$$

where $\boldsymbol{n}_{\boldsymbol{W}}$ is the normal at point $\boldsymbol{x}_{\boldsymbol{W}}$.

For the pressure, two methods are considered. The first approach consists of extrapolating the pressure to the second order using a Taylor expansion similarly to what has been done for velocity:

$$
P_{W}=\bar{P}_{J}+\mathcal{D}_{1}^{*(1)}[\bar{P}]_{J} \cdot\left(x_{W}-x_{j}\right)+O\left(h^{2}\right)
$$

When curvature is taken into account, a second approach more physical can be used, which consists in using the normal momentum equation verified for the Euler equations at the wall [23]:

$$
\frac{\partial P}{\partial n \mid W}_{\mid W}=-\frac{\rho \boldsymbol{u}_{\text {tan }}^{2}}{R}
$$

with $R$ the local radius of curvature of the wall face (which depends on the tangential velocity at the wall point and on the corresponding normal as explained in Section [III, $\boldsymbol{u}_{\boldsymbol{t a n}}$ the tangential velocity magnitude and $\rho$ the density. Eq 34 is discretized using the previous calculation of $\boldsymbol{u}_{\boldsymbol{W}}$ (Eqs. 31 and 32), leading to the second order formula

$$
P_{W}=P_{1}-\frac{\bar{\rho}_{J} u_{W}^{2}}{R}\left\|x_{W}-x_{j}\right\|+O\left(h^{2}\right)
$$

Finally, $\rho_{W}$ is calculated using the gas law.

For the Navier-Stokes equations, the no-slip boundary condition is prescribed for the velocity:

$$
\forall x \in A_{J K_{0}}, u(x)=u_{m e s h} .
$$

This can be directly applied in the discretized boundary condition for velocity. Generally, a Dirichlet-type condition is also given for temperature. Pressure, however, can only be defined at the wall through its gradient. This Neumann boundary condition is changed into an equivalent fictive Dirichlet boundary condition by performing a Taylor expansion of order 2 in the normal direction of point $\boldsymbol{x}_{\boldsymbol{W}}$, as explained in part $\mathrm{A}$

For the scheme with 2-exact reconstruction of the solution, pointwise boundary values of the variables at $\boldsymbol{x}_{\boldsymbol{W}}$ must be known to the third order of accuracy. In a first approach, the same iterative method as previously is used. 
For the Euler equations, the slip condition on velocity leads to

$$
u_{W_{0}}=\bar{u}_{J}+\mathcal{D}_{2}^{*(1)}[\bar{u}]_{J} \cdot\left(x_{W}-x_{j}\right)+\frac{1}{2} \mathcal{D}_{1}^{*(2)}[\bar{u}]_{J} \cdot\left(x_{W}-x_{j}\right)^{\otimes 2}+O\left(h^{3}\right)
$$

with the right-hand side taken at the previous timestep. Then the normal part of this value is removed:

$$
u_{W}=u_{W_{0}}-\left(u_{W_{0}} \cdot n_{W}\right) n_{W} .
$$

For pressure

$$
P_{W}=\bar{P}_{J}+\mathcal{D}_{2}^{*(1)}[\bar{P}]_{J} \cdot\left(x_{W}-x_{j}\right)+\frac{1}{2} \mathcal{D}_{1}^{*(2)}[\bar{P}]_{J} \cdot\left(x_{W}-x_{j}\right)^{\otimes 2}+O\left(h^{3}\right)
$$

For the Navier-Stokes equations, the velocity is again prescribed directly (no-slip condition), and so is the temperature. For the Neumann condition on the pressure, a fictive value can be evaluated by a normal Taylor expansion to the third order, using the normal gradient given by the Neumann boundary condition on the one hand, and the normal component of $\mathcal{D}_{1}^{*(2)}[\bar{P}]_{J}$ at the previous timestep on the other hand.

Note that another possible approach would be to build several differential operators, one for each type of boundary condition, and a corresponding set of correction matrices. This method is however cumbersome and costly (this is even more true when the order $k$ of the $k$-exact scheme increases) and is thus not considered here.

\section{Numerical results}

\section{A. Inviscid flow past a smooth Gaussian bump}

The first case is an inviscid flow through a channel with a Gaussian bump. The inflow Mach number is $M=0.5$ with 0 degree angle of attack. Left and right boundaries are respectively a subsonic inflow, and a subsonic outflow (imposed pressure); upper and lower walls are slip walls. The flow is isentropic, thus entropy is used as an indicator of the accuracy of the simulation, since the analytical solution is unknown.

A mesh convergence study is carried out for the entropy error, using grids of 400, 1600, 6400, 25600 and 102400 cells, corresponding respectively to 40,80,160 320, and 640 wall faces along the bump. Results are provided for both the 1-exact and 2-exact reconstruction schemes, using the baseline solver, the solver with curved boundaries, and the solver with curved boundaries and an enriched discretization stencil at the wall.
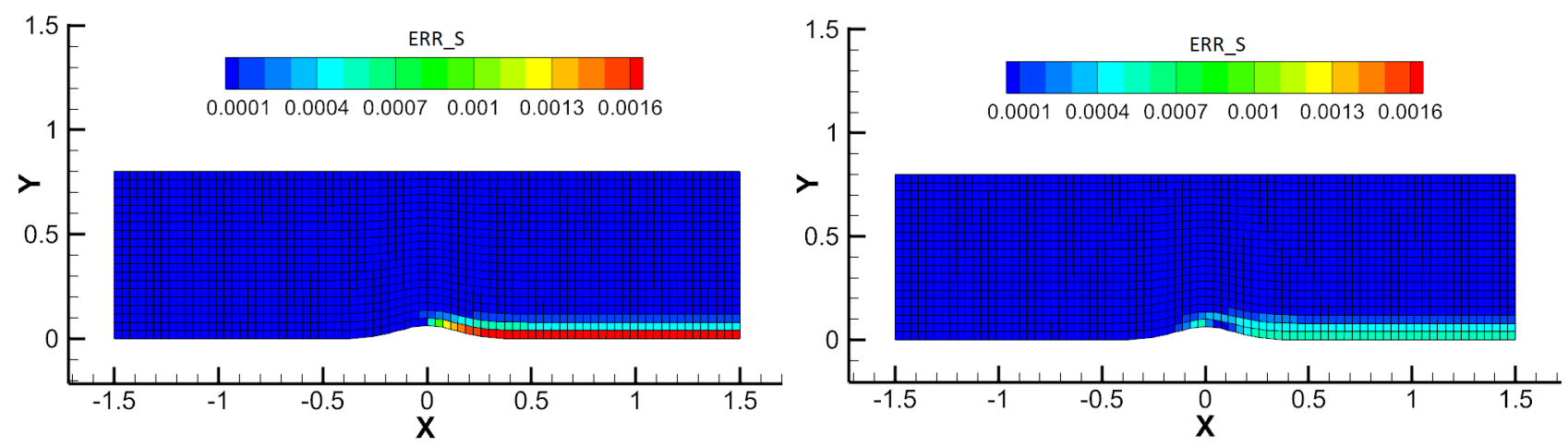

Fig. 3 Inviscid Gaussian bump: entropy error generated downstream of the bump with the 2-exact scheme (a) with no modification; (b) with the wall curvature model

The grid convergence study for the linear reconstruction scheme is shown in Figure 4 using the $L_{2}$ norm.

Firstly, we see that the convergence slope when adding the curvature model to the scheme increases compared to using the unmodified scheme. However, the entropy error reduction is low. This is not surprising: as it was stressed before, the spatial error introduced when approximating curved surfaces with flat faces is of order 2. Yet, the approximation of the boundary should at least represent the real boundary with the same order of accuracy as the discretization scheme [11]. Here the original scheme with 1-exact reconstruction exhibits a convergence of order 2 at most, as can be seen in 
Figure 4. Even if the Bézier patches approximate the wall with third-order accuracy, the gain is expected to be small when this only improvement is used in the solver.

When using the model with extended representation of the solution in boundary cells along with curved walls, much larger improvements are obtained: the entropy error drops and the convergence slope is again increased. The information at the wall is accurate enough thanks to the inclusion of curvature and of a proper handling of boundary conditions (even if the information available at the wall for the Euler equations is limited), and is used consistently to calculate a 1-exact approximation of the gradient.

A grid convergence study is also conducted for the scheme with 2-exact reconstruction (in Figure 5). Here the error is given in $L_{2}$ norm and in $L_{\infty}$ norm. The $L_{2}$ norm measures the average error over the domain, hence giving a global error. On the contrary, the $L_{\infty}$ norm gives the maximum error reached in the domain. In our case, this norm will emphasize the local error in the near-wall region, which is the focal point of this work.

Figure 3 compares the entropy error for the 2-exact reconstruction scheme on the mesh with 80 wall faces along the bump. A generation of numerical entropy is observed downstream of the bump, which is significantly reduced when adding the curvature model. In the rest of the domain where curvature is smaller, entropy is already correctly computed by the baseline method.

Figure 5 shows that very satisfying improvements are obtained with the curvature model, which approximates the physical surface with a spatial error of order 3. The entropy error is reduced and more importantly, the convergence slope is significantly increased with this model (getting closer to third order in $L_{2}$ norm), especially when considering the $L_{\infty}$ norm: this confirms that the improvement of the curved model enhances the scheme near walls. Note that because the $L_{\infty}$ norm is more constraining than the $L_{2}$ norm, the convergence slope obtained with this norm is smaller than the one obtained with the $L_{2}$ norm.

When considering the curvature model coupled with the parabolic reconstruction with a more symmetrical stencil in boundary cells, results are not deteriorated but no significant gain is observed. This may be explained by the fact that the boundary information available at the wall is too limited, and is therefore not sufficient to improve the overall parabolic reconstruction of the solution. To verify this, we test our models on a viscous test case, for which the boundary conditions are better posed than for inviscid flows.

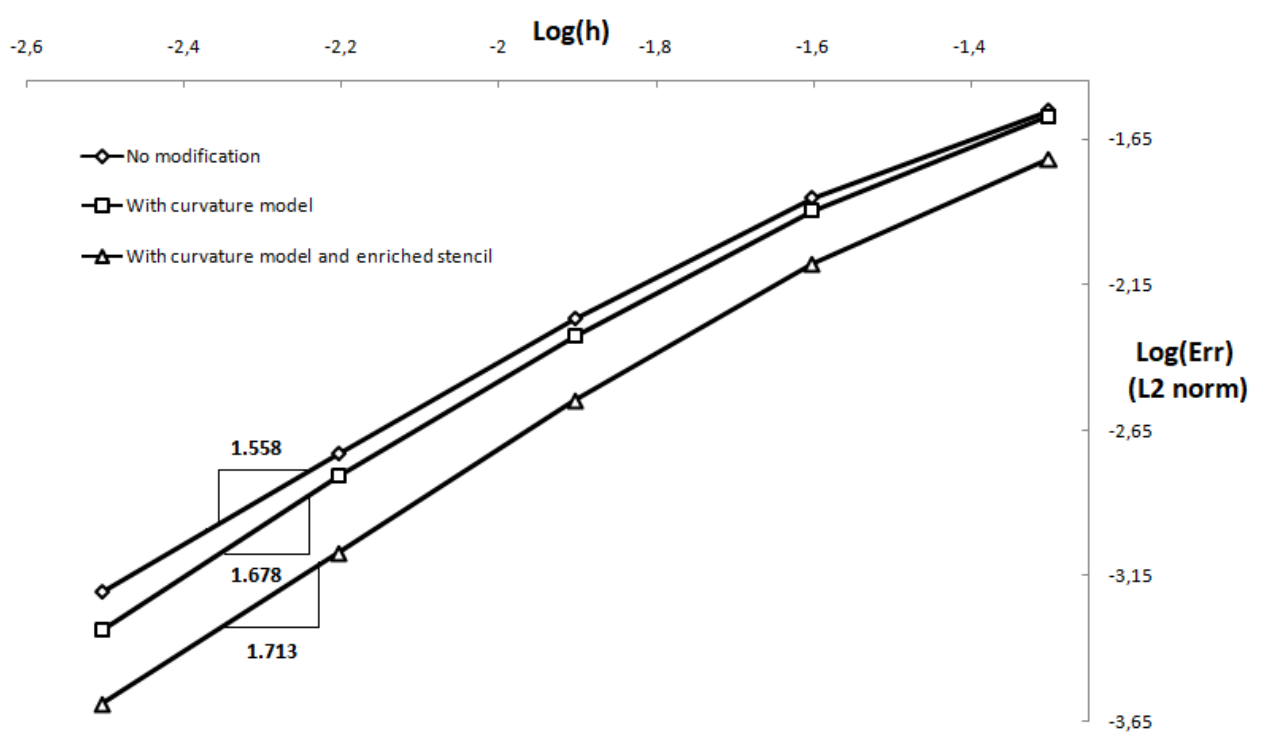

Fig. 4 Mesh convergence on the $L_{2}$ norm of entropy error on the bump test case with the different models, for the 1-exact reconstruction scheme

\section{B. Cylindrical Couette flow}

The chosen viscous test case is the laminar flow in the annulus between two concentric cylinders, with the inner cylinder rotating and the outer cylinder fixed. The radius of the inner cylinder is $R_{i}=1$, and the radius of the outer cylinder is $R_{o}=2$. The inner cylinder is rotated clockwise with a tangential Mach number of $M=0.5$. The viscosity of 


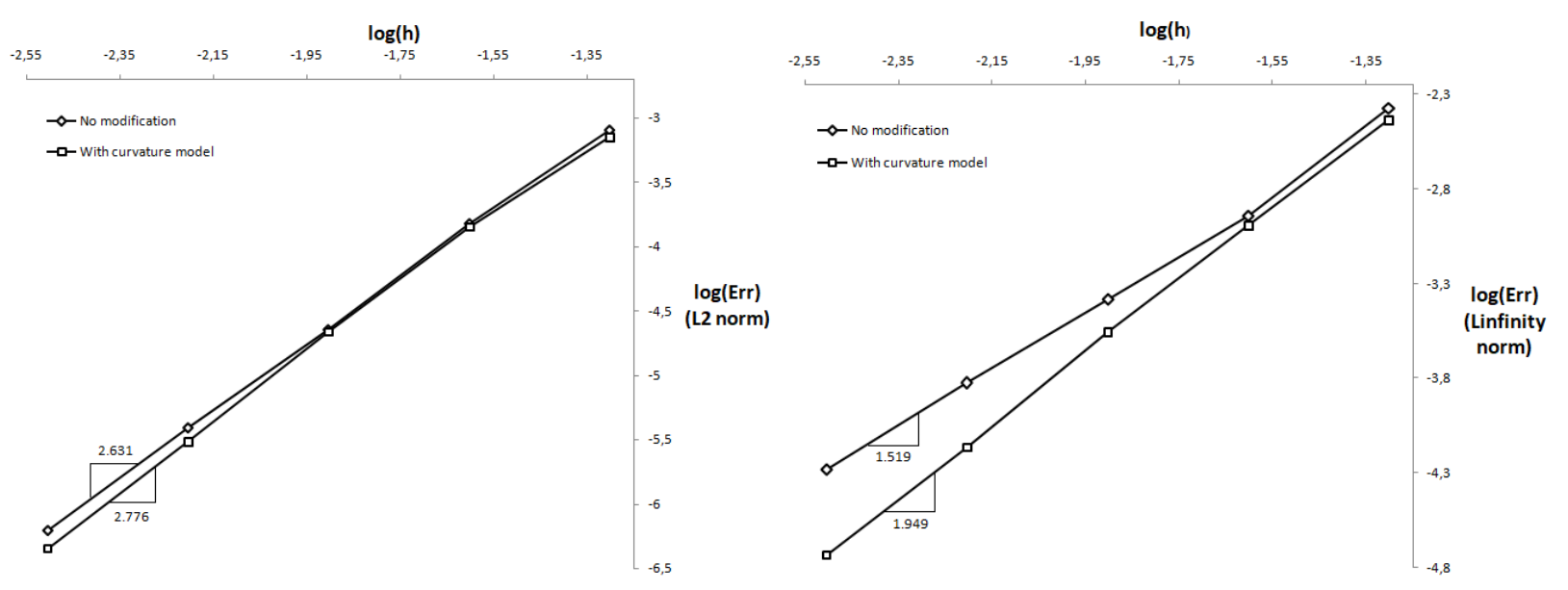

Fig. 5 Grid convergence study showing the reduction of the entropy error on the bump test case with the curved model, for the 2-exact reconstruction scheme. (a) $L_{2}$ norm; (b) $L_{\infty}$ norm

the flow is constant, and a Reynolds number of $R e=10$ based on the inner cylinder radius is chosen as in [24], in order to be sure that the flow does not exhibit toroidal vortices characteristic of the Taylor-Couette instability.

In this stable regime, the flow converges to a steady state for which the velocity is purely azimuthal and can be derived analytically [25]:

$$
u_{\theta, r e f}(r)=\omega_{i} R_{i} \frac{\frac{R_{o}}{r}-\frac{r}{R_{o}}}{\frac{R_{o}}{R_{i}}-\frac{R_{i}}{R_{o}}}
$$

with $\omega_{i}$ the angular velocity and $r$ the radial coordinate in the polar coordinate system.

A mesh convergence study of the error on the azimuthal velocity $E r r_{u_{\theta}}=\frac{\left|u_{\theta}-u_{\theta, r e f}\right|}{\left|u_{\theta, r e f}\right|}$ is performed for a set of five hexahedral grids whose characteristics are presented in the table of Fig. 7 Once again, results are given for the $L_{2}$ and $L_{\infty}$ norms, using the 1-exact (Fig. 8) and 2-exact (Fig. 9p reconstruction schemes. For both schemes, the results are compared for the three models (no modification, with curvature taken into account, with curvature plus enriched stencil close to the wall).

Let us first analyze the results for the scheme with linear polynomial reconstruction. The error on the azimuthal velocity on Grid 2 is presented in Figure 6 , for the original solver with 1-exact reconstruction (left) and for the solver with 1-exact reconstruction coupled with the curvature model and the scheme with enriched stencil at the wall (right). It is clear in this Figure that an important error is generated on the solution close to the wall. This error is locally reduced when the wall curvature is taken into account and the modified reconstruction at boundary cells is introduced.

A grid convergence study is made for the 1-exact scheme is displayed in Figure 8, First-order convergence is observed for all of the compared models, which is due to the fact that diffusive fluxes are in general one order less accurate than convective fluxes.

Figure 8 (b) shows the $L_{\infty}$ error. We see that the scheme coupled with the curvature model produces a lower error than the original scheme. However, the convergence slope appears to be slightly smaller than the one obtained by using the unmodified solver. This might be explained by the fact that the effect of wall curvature corrections is reduced when refining the mesh. Indeed a very good improvement is achieved for the coarsest grid for which the approximation of the wall by flat faces is more detrimental to the scheme.

When the scheme is enriched with this enhanced wall information, the grid convergence shows very good properties. The error is subsequently reduced, and the convergence slope is restored to a value that is actually slightly higher than with the unchanged solver. Notice that for the coarsest grid however, extending the stencil at the wall in addition to the curvature model decreases the performance of the scheme compared to the curvature model alone. This is due to the fact that asymptotical convergence has not been reached yet.

Similar features are revealed by the $L_{2}$ error curves, with less difference observed since the averaged error over all the domain is here displayed. 
Results of the the grid convergence study performed for the scheme with quadratic polynomial reconstruction are reported in Fig. 9 Due to the sensitivity to the viscous fluxes near wall, the expected second-order of accuracy is not retrieved. This reveals the need to provide a special treatment for viscous fluxes, which have not been modified in the present work. However, this does not affect the relative effect of the three models, that will be analyzed in the following. Indeed, the same trends as for the 1-exact reconstruction scheme are observed again for the 2-exact reconstruction scheme asymptotically: the curvature model decreases substantially the error on the azimuthal velocity, but has a slightly smaller order of accuracy; however the correct order of accuracy is restored when the curvature model is used along with the extended stencil.
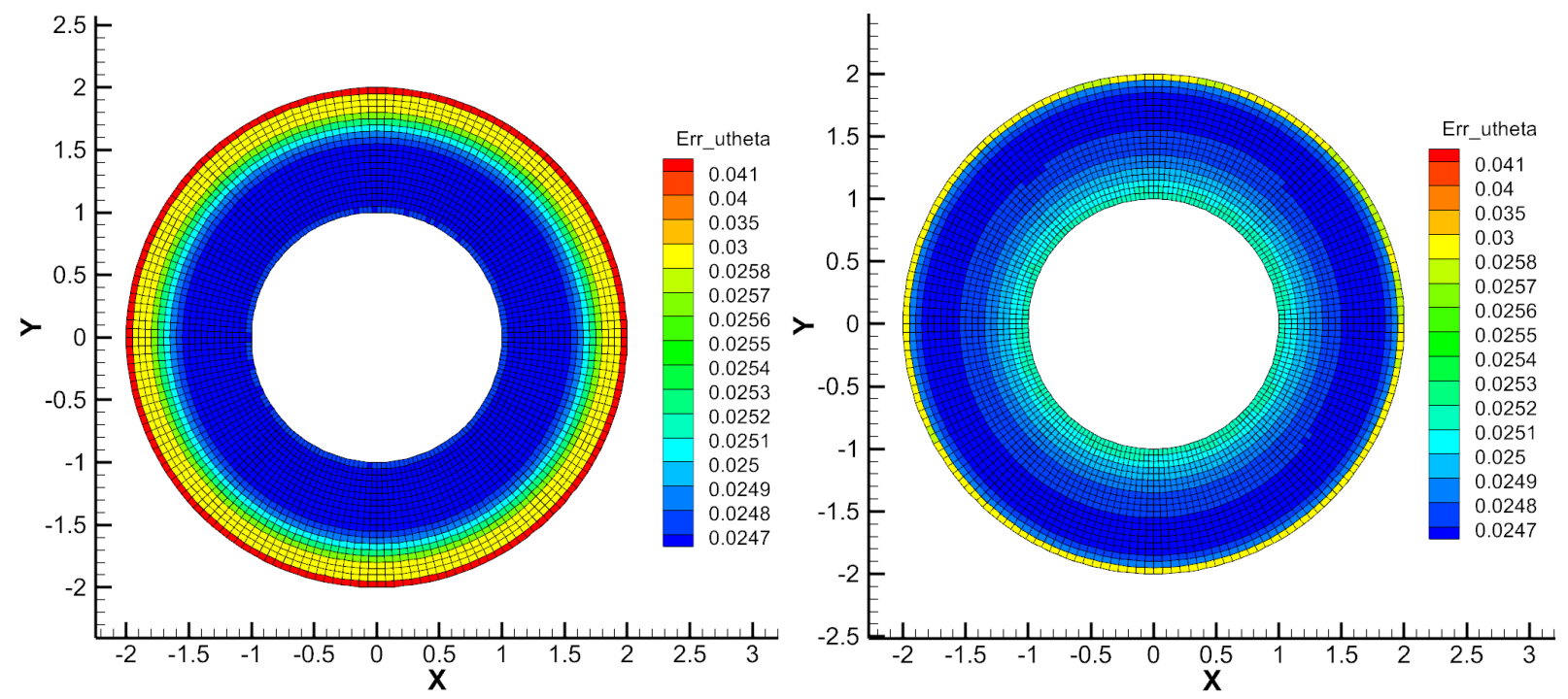

Fig. 6 Plot of the error $\operatorname{Err}_{u_{\theta}}$ on azimuthal velocity for Grid 2, using the 1-exact reconstruction scheme: (a) unmodified solver (b) solver augmented with wall curvature and enriched stencil for bounday cells.

\section{Conclusion and Perspectives}

High-order boundary treatment is investigated for a family of $k$-exact Godunov-type schemes to maintain the order of accuracy in the near-wall region, specifically for configurations with curved boundaries. Two main modifications are proposed to handle the 1-exact and 2-exact reconstruction schemes.

Firstly, a high-order composite representation of the wall based on bicubic Bézier patches is incorporated into the solver. The computation of boundary geometrical quantities is modified to conform to the patches, notably the volume and surface moments, fundamental for the reconstruction and for the fluxes' integration.

Secondly, a numerical strategy is presented for modifying the existing schemes so that the stencil is enriched with the boundary information. For the 1-exact reconstruction scheme, the Quasi-Green operator is extended by adding a wall contribution to the approximation, with a wall pointwise value and a $\beta$ parameter set to 1 , and by modifying the simple correction matrix accordingly. The wall point $\boldsymbol{x}_{\boldsymbol{W}}$ whose normal is colinear to the direction $\boldsymbol{x}_{\boldsymbol{j}}-\boldsymbol{x}_{\boldsymbol{W}}\left(\boldsymbol{x}_{\boldsymbol{j}}\right.$ being the boundary cell centroid) is chosen to evaluate the solution variable. This way, the same methodology can be applied for Dirichlet-type boundary conditions and for Neumann-type boundary conditions, where a fictive Dirichlet value is derived at the wall point by performing a normal Taylor expansion. For the 2-exact scheme, the approximate for the gradient with stencil incorporating the wall is applied only one time in the composition of the operators of the successive corrections procedure. The matrices used to recover consistency are modified in a similar way.

In the proposed procedure, the boundary conditions have to be prescribed at the wall point with at least the same order of accuracy as the reconstruction.

The proposed numerical treatments were first assessed for a compressible inviscid flow past a smooth Gaussian bump. For the 1-exact reconstruction scheme, the improvement due to the curvature model alone is small, but when this is used in conjunction with the extended solver, the entropy is reduced substantially and exhibits a higher convergence slope. This is due to the fact that the error commited when the wall surface is approximated with polygonal faces is 


\begin{tabular}{|ccc|}
\hline Grid & Number of cells & Number of wall faces \\
\hline 0 & 220 & 88 \\
1 & 880 & 176 \\
2 & 3520 & 352 \\
3 & 14080 & 704 \\
4 & 56320 & 1408 \\
\hline
\end{tabular}

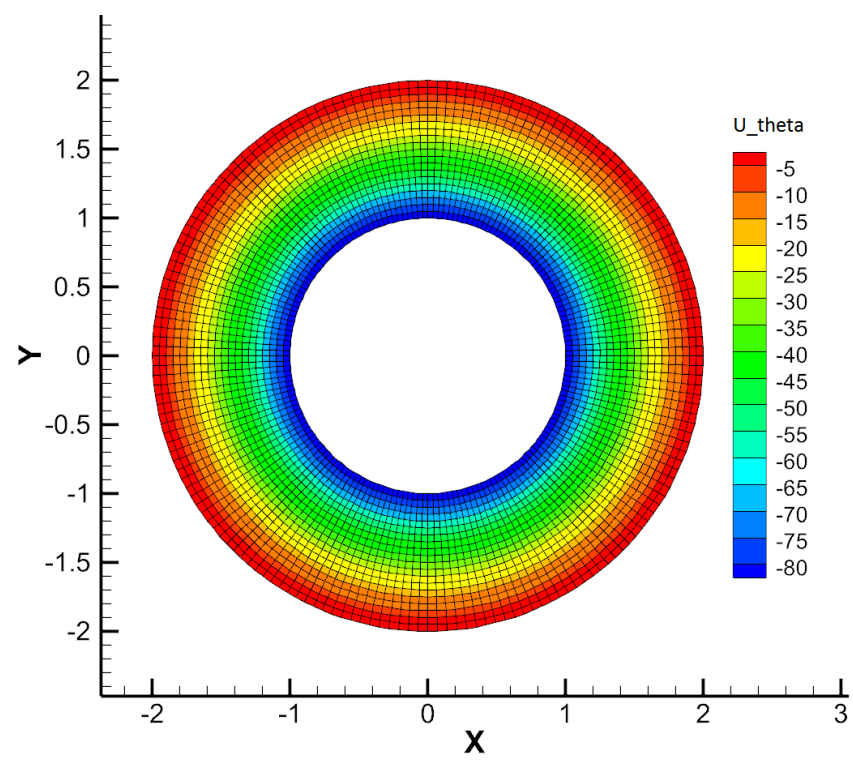

Fig. 7 Circular Couette flow case: (a) description of the meshes used for the grid convergence study; (b) azimuthal velocity $u_{\theta}$ simulated for Grid 2 with the third-order accurate scheme.
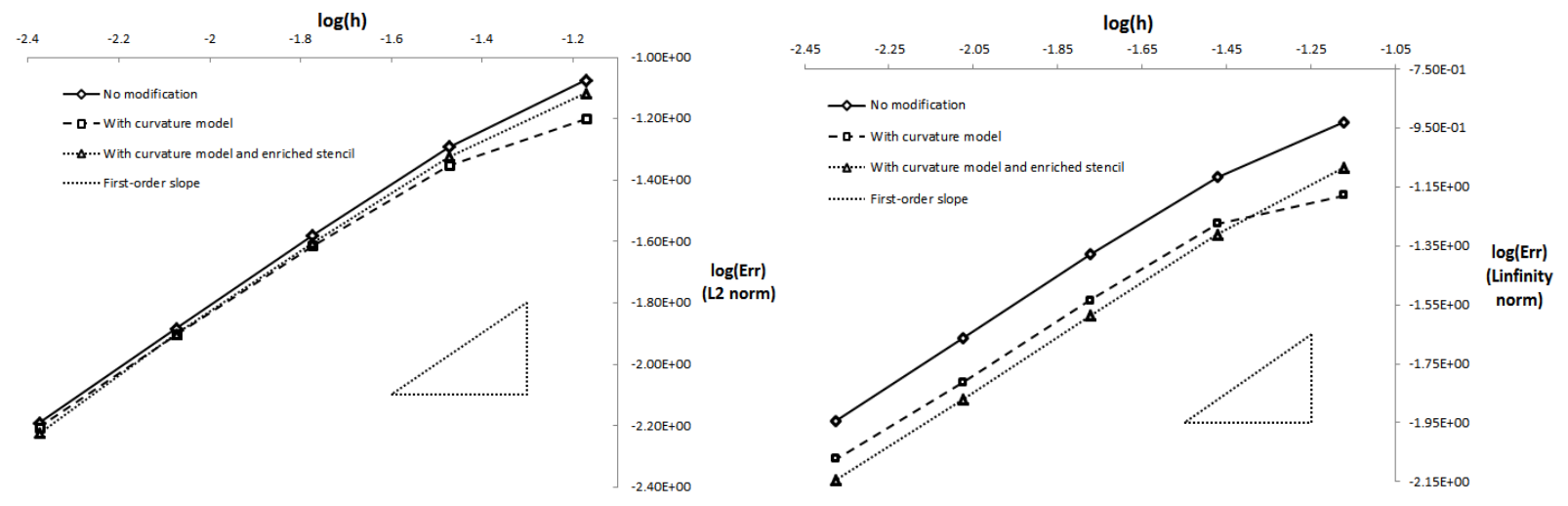

Fig. 8 Grid convergence of the error on the azimuthal velocity for the viscous circular Couette flow: (a) 1-exact reconstruction of the variables, $L_{2}$ norm; (b) 1-exact reconstruction of the variables, $L_{\infty}$ norm.

already of the same order as the numerical scheme itself. On the contrary, when the 2-exact scheme is used, significant increase of the order of accuracy of the method is observed with the curvature model.

In order to test the 2-exact scheme with enriched stencil close to the wall, the laminar Couette flow is then chosen, notably because a true Dirichlet condition is given on the velocity through the no-slip condition. For both schemes, the curvature model decreases the error on the azimuthal velocity. Moroever, the order of accuracy of the method is slightly increased when the curvature model is coupled with the extended stencil versions of the schemes.

These numerical results are encouraging: the modifications are consistent with the existing schemes, and the capability of the method to improve the accuracy of the flow in near-wall regions has been shown. Diffusive fluxes have not been investigated in the present work. However, the integration of these fluxes is directly affected by the reconstruction of the solution and of its derivatives on the one hand, and by the exactness of the geometrical moments on the other hand. Finally, the proposed strategy also leads to improvements for a Couette flow case. Although the diffusive fluxes close to the wall have to be computed more accurately, it does not alter the relative results obtained by using curvature corrections and the enriched stencil. Future work will comprise the modification of the calculation of diffusive fluxes in boundary cells, but also a complete study of low-Mach and low-Reynolds recentring of the scheme in near-wall regions. 


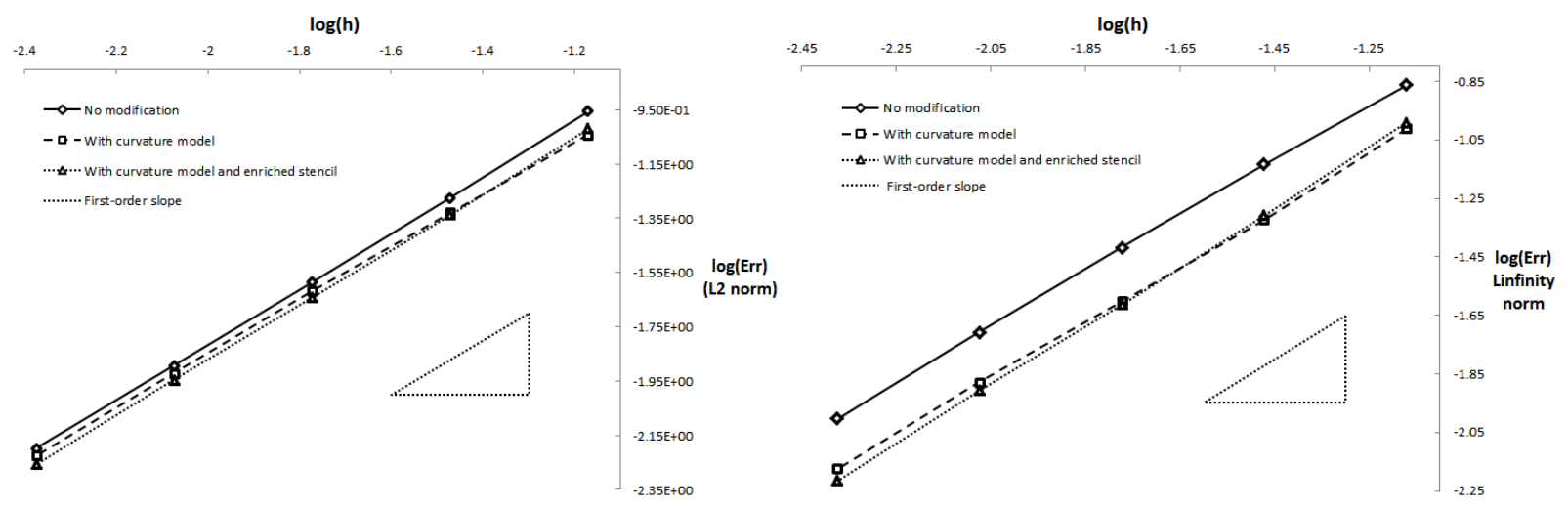

Fig. 9 Grid convergence of the error on the azimuthal velocity for the viscous circular Couette flow: (a) 2-exact reconstruction of the variables, $L_{2}$ norm; (b) 2-exact reconstruction of the variables, $L_{\infty}$ norm.

\section{References}

[1] Barth, T. J., and Frederickson, P. O., "Higher Order Solution of the Euler Equations on Unstructured Grid Using Quadratic Reconstruction," AIAA Paper 90-0013, 1990.

[2] Barth, T. J., "Recent Developments in High Order k-exact Reconstruction on Unstructured Meshes," AIAA Paper 93-0668, 1993.

[3] Vankeirsbilck, P., and Deconinck, H., "Higher order upwind finite volume schemes with ENO-properties for general unstructured meshes," AGARD report, 1992.

[4] Ollivier-Gooch, C., and Van Altena, M., "A High-Order Accurate Unstructured Mesh Finite-Volume Scheme for the AdvectionDiffusion Equation,” Journal of Computational Physics, Vol. 182, No. 2, 2002, pp. 729-752.

[5] Caraeni, D., "Unstructured-Grid Third-Order Finite Volume Discretization Using a Multistep Quadratic Data-Reconstruction Method," AIAA Journal, Vol. 4, 2010, pp. 808-817.

[6] Haider, F., Brenner, P., Courbet, B., and Croisille, J. P., "Efficient Implementation of High Order Reconstruction in Finite Volume Methods," Finite Volumes for Complex Application VI-Problem \& Perspectives, Springer, Vol. 4, 2011, pp. 553-560.

[7] Pont, G. and Brenner, P. and Cinnella, P. and Maugars, B. and Robinet, J. C., "Multiple-correction hybrid k-exact schemes for High-Order compressible RANS-LES simulations on fully unstructured grids," Journal of Computational Physics, Vol. 350, 2017, pp. 45-83.

[8] Brenner, P., "Unsteady flows about bodies in relative motion," Proceedings of the First AFOSR Conference on Dynamic Motion CFD: New Brunswick, New Jersey, 1996.

[9] Qu, F., and Sun, D., "Investigation into the influences of the low-speed flows' accuracy on RANS simulations," Aerospace Science and Technology, Vol. 70, 2017, pp. 578-589.

[10] Giesselmann, J., and Müller, T., "Geometric Error of Finite Volume Schemes for Conservation Laws on Evolving Surfaces," Numerische Mathematik, Vol. 128, No. 3, 2014, pp. 489-516.

[11] Ollivier-Gooch, C., Nejat, A., and Michalak, K., "Obtaining and Verifying High-Order Unstructured Finite Volume Solutions to the Euler Equations," AIAA Journal, Vol. 47, No. 9, 2009, pp. 2105-2120.

[12] Pont, G., Puech, D., Jubera, M., and Brenner, P., "CFD modeling of the Ariane 6 space launch from ground to space," 8th European Symposium on Aerothermodynamics for Space Vehicles, 2015.

[13] Limare, A., Brenner, P., and Borouchaki, H., "Toward a high-order mesh adaptation strategy for unsteady flows on overlapping grids in a finite-volume framework," 46th AIAA Fluid Dynamics Conference, AIAA Aviation Forum, 2016.

[14] Jalali, A., Sharbatdar, M., and Olliver-Gooch, C. F., "Accuracy Analysis of Unstructured Finite Volume Discretization Schemes for Diffusive Fluxes," Computer and Fluids, Vol. 101, 2014, pp. 220-232.

[15] Wang, Q., Ren, Y. X., and Li, W., “Compact high order finite volume method on unstructured grids I: Basic formulations and one-dimensional schemes,” Journal of Computational Physics, Vol. 314, 2016, pp. 863-882. 
[16] Mavriplis, D. J., "Revisiting the Least-Squares Procedure for Gradient Reconstruction on Unstructured Meshes," 16th AIAA Computational Fluid Dynamics Conference, 2003.

[17] Haider, F., Brenner, P., Courbet, B., and Croisille, J. P., “Applications of Efficient Parallel k-exact Finite Volume Reconstruction on Unstructured Grids," ECOMAS Conference in Barcelona, Spain, 2014.

[18] Van Leer, B., "Towards the ultimate conservative difference scheme. V. A second-order Sequel to Godunov method," Journal of Computational Physics, Vol. 32, 1979, pp. 101-136.

[19] Borouchaki, H., "BSHAPE, interpolant quasi G1 d'une surface discrète : descriptif technique," , 2015. Unpublished report.

[20] Bézier, P., The mathematical basis of the UNISURF CAD system, Butterworths, 1986.

[21] Farin, G., Curves and Surfaces for Computer-Aided Geometric Design - A practical guide, Academic Press, Elsevier, 1993.

[22] Walton, D. J., and S., M. D., “A triangular G1 patch from boundary curves,” Computer-Aided Design, Vol. 28, No. 2, 1996, pp. $113-123$

[23] Dadone, A., "Some Solutions of the Equations of Flow of a Viscous Compressible Fluid," International Journal for Numerical Methods in Fluids, Vol. 28, 1998, pp. 1093-1108.

[24] Ollivier-Gooch, C., and Michalak, C., "Unstructured High-Order Accurate Finite-Volume Solutions of the Navier-Stokes Equations," AIAA Paper 2009-954, 47th AIAA Aerospace Sciences Meeting, 2009.

[25] Illingworth, C., "Some Solutions of the Equations of Flow of a Viscous Compressible Fluid," Proc. Cambridge Phil. Soc., Vol. 46, 1950, pp. 469-478. 